\title{
Study of the EAS size spectrum in thermal neutrons measured by the PRISMA-YBJ array
}

\author{
Oleg Shchegolev ${ }^{1, \text { a }}$, Victor Alekseenko ${ }^{1}$, Anastasia Bagrova ${ }^{1,2}$, Yayun $\mathrm{He}^{3}$, Bingbing $\mathrm{Li}^{3}$, Shuwang Cui ${ }^{3}, \mathrm{Xinhua} \mathrm{Ma}^{4}$, \\ Yuri Stenkin $^{1,2}$, and Vladimir Stepanov ${ }^{1}$ \\ ${ }^{1}$ Institute for Nuclear Research, Russian Academy of Sciences, Russia \\ ${ }^{2}$ Nuclear Research National University MEPhI, Russia \\ ${ }^{3}$ College of Physics and Information Engineering, Hebei Normal University, China \\ ${ }^{4}$ Key Laboratory of Particle Astrophysics, Institute of High Energy Physics, Chinese Academy of Sciences, China
}

\begin{abstract}
An Extensive Air Shower array of a novel type has been constructed in Tibet at an altitude of $4300 \mathrm{~m}$ above sea level. It consists of specially designed scintillator en-detectors capable of measuring two main EAS components: hadrons (n) and electrons (e). Results of the experiment are presented. It is demonstrated that the EAS integral distribution in the number of thermal neutrons can be well fitted by a single power law function. Comparison with detailed Monte-Carlo simulations using CORSIKA6.9 and GEANT4.10 codes are also shown.
\end{abstract}

\section{Introduction}

The hadronic component is the main one in Extensive Air Showers (EAS), but is still not studied well due to the absence of inexpensive detectors capable of covering a large area. In 2001 a novel method was developed in INR RAS to solve this problem [1]. The main feature of this method is a new electron-neutron detector (en-detector) capable to simultaneously measure two components of EAS: the electromagnetic component and thermal neutrons produced by the EAS hadronic component. This method allows us to make a large area array of a novel type (PRISMA project). The prototype of this array called PRISMA-YBJ is already running in Tibet (Yangbajing, $4300 \mathrm{~m}$ above sea level) since January 2013. Details of the detector and array can be found elsewhere [2]. The EAS size in thermal neutrons was never studied before, and we present here results obtained after about 3 years of the PRISMA-YBJ continuous operation.

\section{Method}

The array is deployed inside the ARGO-YBJ [3] experimental hall just above RPC plates with a layout shown in Fig. 1. The efficiency of the scintillator for thermal neutron capture was found to be $20 \%$ but the efficiency of the en-detector for thermal neutron recording is $14 \%$ due to pulse height selection. The neutrons are recorded as delayed pulses within a time gate of $20 \mathrm{~ms}$. It is more than enough to collect a great bulk of thermal neutrons produced by EAS hadrons [4]. An on-line program pre-analyzes the data and stores the energy deposit (above a threshold of 2.5 m.i.p.) and the number of recorded neutrons in each detector. A two-fold coincidence trigger condition is applied for

a e-mail: shchegolev_oleg@mail.ru the array to store the data. Additionally, during off-line data processing, 4-fold coincidence with energy deposit above 5 m.i.p. is used for the event selection due to the complicated task of core location.

We applied a traditional maximum-likelihood analysis of EAS, employing the Nishimura-Kamata-Greisen (NKG) function for the electromagnetic component to find the position of the EAS core, its age, EAS size (Ne), etc. As has been shown earlier [5], the total number of secondary neutrons (mostly evaporation ones) is proportional to the number of hadrons passing through the array area. Therefore, measuring the EAS size spectrum in thermal neutrons is equivalent to that in the number of high energy hadrons.

\section{Simulation}

We made a detailed GEANT4-based simulation of our array, including en-detectors and experimental hall. The models we used are QGSP, BIC and NeutronHP. This set is often used in GEANT4 simulations of experiments with neutrons, for example neutron monitors [6]. It allows us to calculate neutron production by EAS hadrons and the neutron thermalization process. The following results were obtained due to the simulations: energy deposit in the en-detector as a function of energy of EAS particles, dependence of neutron multiplicity on EAS hadrons energy and lateral distribution of recorded neutrons around the hadron trajectory [7]. The CORSIKAbased Monte-Carlo simulation uses dependences obtained with GEANT4 to simulate our experiment. The models used in CORSIKA are QGSJET-II.03 and GHEISHA2002. Simulations were performed for primary protons and iron in the energy range $30 \mathrm{TeV}$ (100 $\mathrm{TeV}$ for iron) $300 \mathrm{PeV}$. The integral primary energy spectra index was $=-1.7$ in the whole range. Simulated data are stored at the same format as the experimental ones. 


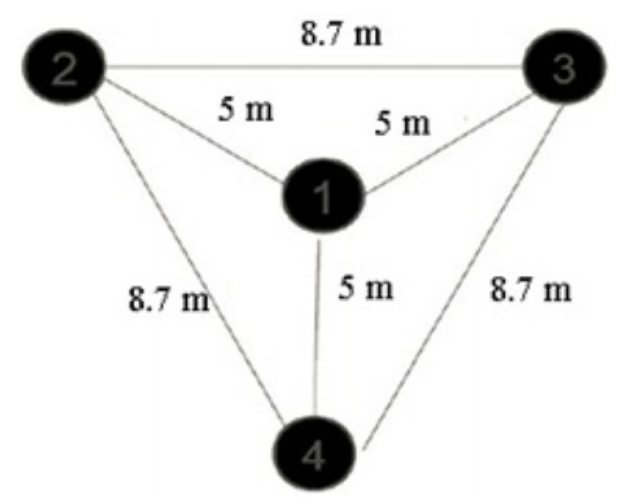

Figure 1. PRISMA-YBJ array layout.

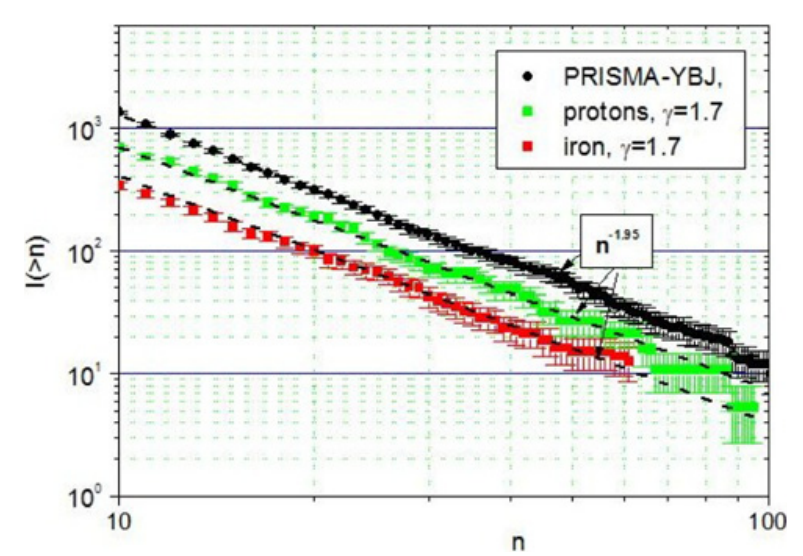

Figure 2. The integral neutron EAS size distribution. Black points experimental data from PRISMA-YBJ array. Red points simulation using CORSIKA and GEANT4 codes for primary protons. Green points the same for primary iron. Curves are shifted to prevent data overlapping

\section{Results}

The experimental integral distribution of showers on the number of recorded thermal neutrons as measured by the PRISMA-YBJ array with en-detectors is shown in Fig. 2 (black points). The spectrum follows a power law with an index of $-1.95 \pm 0.05$ in the range from 10 to 100 neutrons per shower.
As simulations show (Fig. 2, green and red points), the integral EAS distribution on the number of thermal neutrons also follows a power law with index $-1.95 \pm$ 0.05 . Results of the experiment and our simulations are in good agreement.

\section{Conclusion}

A prototype of a novel type of EAS experiment (PRISMAYBJ) is running in Tibet for more than 3 years. It is noticeable that, for the first time, the EAS size spectrum in thermal neutrons at mountain level measured with the array shows a pure power law behavior in the truncated neutron multiplicity range corresponding to cosmic ray primary energies 1-10 PeV. This result is in excellent agreement with that obtained by us at sea level [8].

This study was performed at the Institute for Nuclear Research Russian Academy of Sciences. The Computational Cluster of the Theoretical Department of INR RAS was used for simulations. This work was supported in Russia by RFBR (grants 14-0200996, 16-32-00054 and 16-29-13067_ofi_m), RAS Praesidium Program "Fundamental properties of matter and astrophysics", and in China by NSFC (No. 10975046, No. 11375052).

\section{References}

[1] Yu.V. Stenkin and J.F. Valdes-Galicia, Proc. of 27th ICRC, Hamburg 4, 326 (2001)

[2] B. Bartoli et al., Astropart. Phys. 81, 49-60 (2016)

[3] G. Staiti et al., Nucl. Instr. Meth. Phys. Res. A, 588, 1-2, 7-13 (2008)

[4] Yu.V. Stenkin, Nucl. Phys. B (Proc. Suppl.) 196, 293-296 (2009)

[5] Yu.V. Stenkin et al., Chinese Physics C, 37, 015001 (2013)

[6] P. Paschalis et al., New Astronomy, 33, 26-37 (2014)

[7] O.B. Shchegolev et al., Journ. of Phys.: Conf. Ser. 718, 052038 (2016)

[8] A.A. Petrukhin et al., Proc. 34th ICRC, Hague, PoS(ICRC2015), 427 (2015) 